\title{
Caracterización morfológica de la cabra Motilona de Norte de Santander, Colombia
}

\author{
William Herrera-Cáceres ${ }^{1 *} \bowtie\left(\mathbb{0} ;\right.$; Leonardo Hernández-Corredor ${ }^{1,2} \bowtie(\mathbb{0}$.
}

\begin{abstract}
${ }^{1}$ Universidad Francisco de Paula Santander, Facultad de ciencias agrarias y del ambiente, Cúcuta, Colombia. 2Servicio Nacional de Aprendizaje SENA - Regional Norte de Santander, Cúcuta, Colombia.

*Correspondencia: williamandresh@hotmail.com
\end{abstract}

Recibido: Septiembre 2020; Aceptado: Junio 2021; Publicado: Noviembre 2021.

\section{RESUMEN}

Objetivo: Describir las características morfológicas de la cabra Motilona de Norte de Santander. Material y métodos: Se utilizó una población de 300 individuos (266 hembras y 34 machos) que fueron seleccionadas de forma aleatoria en 8 granjas diferentes del departamento Norte de Santander. La caracterización morfológica de la cabra Motilona se hizo mediante información de 7 rasgos morfológicos cualitativos y 11 rasgos cuantitativos de los cuales se derivaron 14 índices zoométricos. Resultados: Se encontró que existe dimorfismo sexual en los rasgos cualitativos de tipo de cuernos, posición de las orejas y el número de capas de color. Se encontró también que existe dimorfismo sexual entre rasgos cuantitativos como longitud de la cara, anchura de la cabeza, alzada de la cruz, anchura de la grupa, perímetro de la caña y perímetro del tórax. Conclusiones: Los resultados de este estudio sugieren que la cabra Motilona es una población con rasgos homogéneos y por lo tanto se requiere de futuros estudios para profundizar en el conocimiento de caracterización de otros rasgos morfológicos y además en sus características productivas.

Palabras clave: Cabras; morfología animal; programas de conservación (Fuente: CAB).

\section{ABSTRACT}

Objective: To describe the morphological traits of the Motilona goat from Norte de Santander. Material and methods: A population of 300 individuals (266 females and 34 males) was used, which were randomly selected from 8 different farms in the Norte de Santander. The morphological characterization of the Motilona goat was made using information from 7 qualitative morphological traits and 11 quantitative traits from which 14 zoometric indices were derived. Results: Sexual dimorphism was observed in the qualitative traits of horn type, ear position and the number of color layers. Sexual dimorphism was also observed between quantitative traits such as length of the face, width of the head, height of the cross, width of the rump, perimeter of the cane and perimeter of the thorax. Conclusions: The results of this study suggest that the Motilona goat is a population with homogeneous traits and therefore future studies are required to deepen the knowledge of characterization of other morphological traits and in their productive traits.

Keywords: Goats; animal morphology; conservation programs (Fuente: $C A B$ ).

Como citar (Vancouver).

Herrera-Cáceres W, Hernández-Corredor L. Caracterización morfológica de la cabra Motilona de Norte de Santander, Colombia. Rev MVZ Córdoba. 2022; 27(1):e2149. https://doi.org/10.21897/rmvz.2149 


\section{INTRODUCCIÓN}

En la región de Norte de Santander (Colombia) la cría del caprino se lleva a cabo principalmente para la producción de carne y leche para consumo humano. Esta actividad ganadera que ha venido en aumento en los últimos años no solo en la región sino también en Colombia $(1,2)$, requiere mayor atención para mejorar los sistemas de producción. Para esto, caracterizar las poblaciones de cada región es indispensable para tener un inventario de los recursos animales con los que se cuenta (3), esto permitirá, por un lado, emplear de forma sostenible estos recursos animales para abastecer a una población en continuo crecimiento y por otro, conservar los recursos zoogenéticos (4). Esto se consigue con la caracterización morfológica, la cual es una alternativa comúnmente utilizada para profundizar en el conocimiento de poblaciones caprinas desconocidas $(3,4,5,6,7,8)$.

La caracterización racial de una especie es importante para la conservación de esta, pero en el contexto de la producción animal, esta caracterización es relevante para implementar programas de mejora genética (3). Esta caracterización se realiza a través de medidas zoométricas, las cuales son consideradas como variables morfoestructurales que se obtienen a partir del fenotipo de los animales y, junto con las características fanerópticas, también son todas de origen genético y sirven como marcadores externos para medir aquellas características de importancia económica en producción animal $(9,10,11)$. Por otra parte, el estudio de las mediciones corporales en vivo de un animal permite llegar a obtener un grupo racial con una conformación definida para un fin zootécnico, por ejemplo, establecer el grado de asociación de una determinada medida corporal con alguna característica de interés productivo como puede ser la estimación del peso de la carne (7). El objetivo de este estudio es caracterizar la estructura morfológica de la cabra Motilona para determinar su propósito productivo y para definir sus características fenotípicas.

\section{MATERIAL Y MÉTODOS}

Población de estudio. Para este estudio se seleccionaron individuos de diferentes localidades de la ciudad de Cúcuta y su área metropolitana. De esta manera se garantizó que los individuos de la población muestreada pertenecieran todos a las mismas condiciones climáticas de una región geográfica que se encuentra a una altura de 320 MSNM, con un rango de temperatura promedio a lo largo del año de $22-36^{\circ} \mathrm{C}$ y un rango de humedad relativa promedio durante el año de 40-95\%. Entre los años 2013 y 2016, se visitaron 8 granjas para recoger los datos de las hembras y 7 para los machos. Se recolectó información para un total de 300 animales: 266 hembras y 34 machos. Estos animales tenían una dieta a base de hierba que adquirían durante el periodo de pastoreo, que empezaba alrededor de las $7 \mathrm{~h}$ y finalizaba cerca de las $18 \mathrm{~h}$. Con respecto a la hierba, esta se basaba principalmente de rastrojos obtenidos de diferentes especies arbóreas de la región entre las que se encuentran algunas como Pardillos, Urapos o Yátagos. De modo que durante el día estaban afuera comiendo y durante la noche eran guardados nuevamente en los corrales. Este sistema de alimentación les permitía, a los criadores, llevar a cabo inspecciones periódicas relacionadas con el estado de salud y la condición corporal de los animales durante la noche.

Evaluación fenotípica. Para evaluar las características fenotípicas de la cabra Motilona se tomaron registros morfológicos de machos jóvenes que tenían descendientes en la población. En hembras, se tomaron registros morfológicos de aquellos individuos que se encontraban en periodo de lactancia después de haber tenido su primer o segundo parto. De esta forma se recolectaron registros morfológicos de animales que se encontraban en etapa productiva y que habían alcanzado su madurez sexual. Empleando un bastón zoométrico y una cinta métrica se midieron 11 variables cuantitativas que son comúnmente utilizadas para la caracterización morfológica del caprino $(3,8)$ : longitud de la cabeza (LC), longitud de la cara (LR), anchura de la cabeza (AC), alzada de la cruz (ALCR), anchura de grupa (AG), diámetro longitudinal $(D L)$, diámetro dorso esternal (DE), diámetro bicostal (DB), longitud de grupa (LG), perímetro de la caña (PC) y perímetro del tórax (PT). A partir de estas medidas se derivaron 14 índices zoométricos: Índice corporal (ICO=DLx100/ $\mathrm{PT})$, índice torácico (ITO $=\mathrm{DB} \times 100 / \mathrm{DE})$, índice cefálico (ICE $=A C \times 100 / L C)$, índice pelviano (IPE $=A G \times 100 / L G)$, índice de proporcionalidad (IPRO=DLx100/ALCR), índice metacarpotorácico (IMETO=PCx100/PT), índice metacarpocostal (IMCOS $=P C \times 100 / D B$ ), índice de profundidad relativa del tórax (IPRP $=\mathrm{DEx} 100 / \mathrm{ALCR})$, índice pelviano transversal (IPET=AGX100/ALCR), índice pelviano longitudinal (IPEL $=L G \times 100$ / ALCR), índice de compacidad (ICOMP $=\mathrm{P} \times 100 /$ ALCR) e índice de espesor relativo de la caña (IERCAÑ =PCx100/ALCR). Además de estas variables cuantitativas, se midió también el 
peso vivo de los animales $(P)$ mediante una cinta de pesaje. Se realizó un análisis de varianza (ANOVA) para comparar las medias post hoc entre localidades y también entre sexos con la prueba $\mathrm{F}$ múltiple de Ryan-EinotGabriel-Welsch (12). Las variables cuantitativas se agruparon y se relacionaron mediante un análisis de componentes principales (ACP). Las cargas factoriales fueron rotadas con el método Varimax, para minimizar las variables que presenten saturaciones altas en cada componente (13). Adicional a esto, se realizó un análisis discriminante (14) para explorar cómo se clasifican los animales entre sexos.

Análisis estadístico. Para el estudio de los rasgos fanerópticos (8), se midieron 7 variables cualitativas: el tipo de cuernos, presencia de mamellas, línea dorso-lumbar, posición de las orejas, inclinación de la grupa, angulosidad y el número de capas del pelo. Para estas variables se calcularon las frecuencias absolutas y relativas, junto con pruebas estadísticas de significación de chi-cuadrado $\left(X^{2}\right)$ para el contraste entre sexos, también se realizó un análisis de correspondencia múltiple (ACM) para describir la dependencia de las variables y sus posibles relaciones (15). Este método de ACM, así como el ACP y el análisis discriminante mencionados anteriormente, fueron realizados con el programa estadístico SPSS v. 20 (SPSS inc, Chicago, Illinois, USA).

\section{RESULTADOS}

En la tabla 1 se encuentran los valores de los estadísticos descriptivos calculados para cada uno de los rasgos cuantitativos y los índices zoométricos derivados a partir de estos. Los resultados de las variables cuantitativas mostraron que existe dimorfismo sexual en la población para las variables $L C, L R, A C, A L C R$, $\mathrm{DE}, \mathrm{PC}$ y $\mathrm{PT}$, junto con los índices corporales IPRO, IMCOS, IPET, ICOMP e IERCAÑ porque estas resultaron tener diferencias significativas entre sexos $(p<0.05)$. Con respecto a las diferentes localidades donde estaba distribuida la población de hembras bajo estudio, se encontraron diferencias significativas en todas las variables $(p<0.05)$, excepto $L G, P C, P T$, IPRO e IPRP. El coeficiente de variación (CV) para las variables cuantitativas de las hembras y los machos estuvo entre 4.90-16.48 y entre 8.05-34.37, respectivamente. Mientras que el CV de los índices corporales en hembras y machos estuvo entre 5.87-19.25 y entre 6.70-28.58, respectivamente.

Tabla 1. Media (M), desviación típica (DT) y coeficiente de variación (CV) de las variables cuantitativas y de los índices zoométricos de la cabra Motilona.

\begin{tabular}{|c|c|c|c|c|c|c|c|c|c|}
\hline \multirow{2}{*}{ Variables } & \multicolumn{3}{|c|}{ Hembras $(n=266)$} & \multicolumn{3}{|c|}{ Machos $(n=34)$} & \multirow{2}{*}{ Sexos } & \multicolumn{2}{|c|}{ Granjas } \\
\hline & $\mathbf{M}$ & DT & CV(\%) & $\mathbf{M}$ & DT & CV(\%) & & Hembras & Machos \\
\hline $\mathrm{P}, \mathrm{kg}$ & 44.63 & 7.36 & 16.49 & 55.56 & 19.10 & 34.37 & * & * & n.s. \\
\hline $\mathrm{LC}, \mathrm{cm}$ & 23.98 & 1.60 & 6.67 & 25.66 & 2.69 & 10.48 & $*$ & * & n.s. \\
\hline $\mathrm{LR}, \mathrm{cm}$ & 14.50 & 1.32 & 9.10 & 15.15 & 2.20 & 14.52 & * & $*$ & * \\
\hline$A C, \mathrm{~cm}$ & 12.91 & 0.85 & 6.58 & 13.54 & 1.45 & 10.70 & * & * & n.s. \\
\hline $\mathrm{ALCR}, \mathrm{cm}$ & 68.94 & 3.38 & 4.90 & 74.10 & 5.97 & 8.05 & $*$ & $*$ & n.s. \\
\hline $\mathrm{AG}, \mathrm{cm}$ & 17.66 & 1.26 & 7.13 & 17.77 & 1.87 & 10.52 & n.s. & $*$ & $*$ \\
\hline $\mathrm{DL}, \mathrm{cm}$ & 63.66 & 4.09 & 6.42 & 64.28 & 10.47 & 16.29 & n.s. & $*$ & $*$ \\
\hline $\mathrm{DE}, \mathrm{cm}$ & 30.36 & 2.20 & 7.25 & 32.69 & 4.59 & 14.04 & $*$ & $*$ & n.s. \\
\hline $\mathrm{DB}, \mathrm{cm}$ & 11.88 & 1.69 & 14.23 & 12.03 & 2.05 & 17.04 & n.s. & $*$ & $*$ \\
\hline $\mathrm{LG}, \mathrm{cm}$ & 16.17 & 1.11 & 6.86 & 16.51 & 1.72 & 10.42 & n.s. & n.s. & n.s. \\
\hline $\mathrm{PC}, \mathrm{cm}$ & 13.98 & 1.28 & 9.16 & 15.50 & 1.29 & 8.32 & $*$ & n.s. & n.s. \\
\hline $\mathrm{PT}, \mathrm{cm}$ & 79.54 & 5.25 & 6.60 & 82.65 & 8.58 & 10.38 & $*$ & n.s. & n.s. \\
\hline ICO & 80.24 & 5.52 & 6.88 & 77.99 & 11.44 & 14.67 & n.s. & $*$ & $*$ \\
\hline ITO & 38.67 & 5.51 & 14.04 & 37.24 & 6.74 & 18.10 & n.s. & * & n.s. \\
\hline ICE & 54.01 & 4.40 & 8.15 & 52.87 & 3.72 & 7.03 & n.s. & $*$ & $*$ \\
\hline IPE & 109.51 & 7.85 & 7.17 & 108.47 & 13.60 & 12.54 & n.s. & $*$ & n.s. \\
\hline IPRO & 92.42 & 5.42 & 5.87 & 86.75 & 12.15 & 14.00 & $*$ & n.s. & * \\
\hline IMETO & 17.64 & 1.81 & 10.25 & 18.83 & 1.26 & 6.70 & n.s. & $*$ & n.s. \\
\hline IMCOS & 119.46 & 16.13 & 13.51 & 131.86 & 21.06 & 15.97 & $*$ & * & $*$ \\
\hline IPRP & 44.10 & 3.37 & 7.63 & 44.10 & 4.94 & 11.21 & n.s. & n.s. & n.s. \\
\hline IPET & 25.65 & 1.83 & 7.15 & 24.03 & 2.17 & 9.01 & $*$ & $*$ & $*$ \\
\hline IPEL & 23.48 & 1.61 & 6.87 & 23.30 & 1.79 & 8.03 & n.s. & * & n.s. \\
\hline ICOMP & 64.65 & 9.58 & 14.82 & 73.99 & 21.15 & 28.58 & $*$ & * & $*$ \\
\hline IERCAÑ & 20.30 & 1.70 & 8.39 & 20.99 & 1.77 & 8.45 & * & * & n.s. \\
\hline
\end{tabular}

P: peso vivo de los animales, LC: longitud de la cabeza, LR: longitud de la cara, AC: anchura de la cabeza, ALCR: alzada de la cruz, AG: anchura de la grupa, DL: diámetro longitudinal, DE: diámetro dorso esternal, DB: diámetro bicostal, LG: longitud de la grupa, PC: perímetro de la caña, PT: perímetro del tórax, ICO: índice corporal, ITO: índice torácico, ICE: índice cefálico, IPE: índice pelviano, IPRO: índice de proporcionalidad, IMETO: índice metacarpotorácico, IMCOS: índice metacarpocostal, IPRP: índice de profundidad relativa del tórax, IPET: índice pelviano transversal, IPEL: índice pelviano longitudinal, ICOMP: índice de compacidad, IERCAÑ: índice de espesor relativo de la caña. CV: coeficiente de variación, DT: desviación típica. *Diferencias estadísticamente significativas $(p<0.05)$, n.s.: no existen diferencias significativas. 
El análisis de componentes principales rotados para la cabra Motilona mostró que las variables que se asocian de acuerdo con la matriz de rotación son ALCR, AG, DL, DE, LG, PC y PT de la componente uno con LC, LR y AC de la componente dos, y también con $A G$ y $D B$ de la componente tres. Estas tres componentes explicaron el $62.5 \%$ de la varianza total. Un punto importante de estos resultados es que la componente uno se encontró relacionada con aquellas medidas que fueron tomadas en el tórax y las extremidades de los animales, mientras que la componente dos se encontró relacionada con medidas que fueron tomadas en la cabeza de los animales (Tabla 2 ). Por otro lado, los resultados del análisis discriminante mostraron que $P$, ALCR, DE y PC fueron las variables que mejor discriminaron entre ambos sexos (Tabla 3).

Tabla 2. Matriz de componentes principales rotados para las variables cuantitativas medidas en los 300 individuos.

\begin{tabular}{cccc}
\hline Variables & Componente 1 Componente 2 Componente 3 \\
\hline LC & 0.476 & 0.587 & 0.269 \\
LR & 0.105 & 0.826 & 0.242 \\
AC & 0.193 & 0.794 & -0.211 \\
ALCR & 0.636 & 0.401 & 0.131 \\
AG & 0.627 & 0.205 & -0.510 \\
DL & 0.692 & 0.190 & 0.022 \\
DE & 0.606 & 0.182 & 0.226 \\
DB & 0.211 & 0.163 & 0.802 \\
LG & 0.781 & 0.135 & -0.039 \\
PC & 0.638 & -0.016 & 0.419 \\
PT & 0.742 & 0.188 & 0.123 \\
$\% V$ & 40.874 & 11.099 & 10.572 \\
\hline
\end{tabular}

$\mathrm{KMO}=0.856$. Esfericidad de Bartlett $\mathrm{p}<0.001$. LC: longitud de la cabeza, LR: longitud de la cara, AC: anchura de la cabeza, ALCR: alzada de la cruz, AG: anchura de la grupa, DL: diámetro longitudinal, DE: diámetro dorso esternal, DB: diámetro bicostal, LG: longitud de la grupa, PC: perímetro de la caña, PT: perímetro del tórax, \%V: Porcentaje de varianza.

Los resultados de los rasgos cualitativos son presentados en la tabla 4, donde se observó también un dimorfismo sexual entre machos y hembras para las variables: tipo de cuernos, la inclinación de la grupa, la angulosidad corporal y el número de capas. En el caso del tipo de cuernos, los machos tienden a tener, en la mayoría de los casos, cuernos cortos y cuernos en forma de espiral (41\% y 32\%, respectivamente), mientras que la mayoría de la población de hembras $(63 \%)$ presentaron cuernos curvos y solo el $26 \%$ de las hembras estudiadas tenían cuernos cortos $(p<0.001)$.
Tabla 3. Porcentaje de animales que clasifican bien para el sexo según la variable evaluada de acuerdo con los resultados del análisis discriminante para los 300 individuos.

\begin{tabular}{cccccc}
\hline Variables H (\%) & M (\%) & PD (\%) & $\begin{array}{c}\text { Prueba } \\
\text { M de Box }\end{array}$ & $\begin{array}{c}\text { Prueba } \\
\text { Lambda } \\
\text { de Wiks }\end{array}$ \\
\hline P & 76.7 & 47.1 & 73.3 & $*$ & $*$ \\
LC & 69.9 & 55.9 & 68.3 & $*$ & $*$ \\
LR & 61.3 & 55.9 & 60.7 & $*$ & $*$ \\
AC & 68.0 & 52.9 & 66.3 & $*$ & $*$ \\
ALCR & 78.2 & 58.8 & 76.0 & $*$ & $*$ \\
AG & 52.3 & 44.1 & 51.3 & $*$ & n.s. \\
DL & 56.0 & 50.0 & 55.3 & $*$ & n.s. \\
DE & 74.8 & 55.9 & 72.7 & $*$ & $*$ \\
DB & 51.5 & 52.9 & 51.7 & n.s. & n.s. \\
LG & 56.0 & 47.1 & 55.0 & $*$ & n.s. \\
PC & 86.1 & 73.5 & 84.7 & n.s. & $*$ \\
PT & 64.7 & 47.1 & 62.7 & $*$ & $*$ \\
P+ALCR+ & 82.0 & 61.8 & 79.7 & $*$ & $*$ \\
DE+PC & & & & $*$ & $*$ \\
ALCR+PC & 81.6 & 67.6 & 80.0 & $*$ & $*$
\end{tabular}

P: peso vivo, LC: longitud de la cabeza, LR: longitud de la cara, AC: anchura de la cabeza, ALCR: alzada de la cruz, AG: anchura de la grupa, DL: diámetro longitudinal, DE: diámetro dorso esternal, DB: diámetro bicostal, LG: longitud de la grupa, PC: perímetro de la caña, PT: perímetro del tórax. H: hembras, M: machos, PD: porcentaje de los 300 animales que clasifican bien para su respectivo sexo según la variable evaluada. ${ }^{*} p<0.05$, n.s.: no existen diferencias significativas.

En cuanto al rasgo de inclinación de la grupa, la mayor parte de la población de hembras $(66.5 \%)$ tenían una inclinación recta, mientras que la mitad de los machos muestreados (50\%) tenían una grupa muy inclinada y el $44 \%$ de los machos tenían una grupa poco inclinada $(p<0.001)$. En cuanto a la angulosidad corporal, los machos presentaron una tendencia a ser de angulosidad intermedia a ancha, mientras que en las hembras se observó lo contrario, angulosidad intermedia a delgada $(p<0.001)$. En cuanto al número de capas de hembras y machos, ambos tuvieron una tendencia a tener entre 2 y 3 capas de colores diferentes, sin embargo, el porcentaje de hembras muestreadas con 3 capas fue de $51.5 \%$ y hembras con 2 capas fue de $33.8 \%$, mientras que en machos estos porcentajes fueron de $23.5 \%$ y $41 \%$, respectivamente $(p<0.001)$. En cuanto a la posición de las orejas en las hembras, el $97.7 \%$ de la población mostró tener unas orejas alargadas que cuelgan, mientras que en machos se observó que el $73.5 \%$ tenía este tipo de orejas, pero además el $26.4 \%$ de los machos tenían también orejas en posición horizontal $(p<0.001)$. 
Herrera-Cáceres y Hernández - Corredor Caracterización morfológica de la cabra Motilona

Tabla 4. Frecuencias absolutas y frecuencias relativas de los caracteres cualitativos medidos en la cabra Motilona.

\begin{tabular}{|c|c|c|c|c|c|c|}
\hline \multirow{2}{*}{ Rasgo } & \multirow{2}{*}{ Categoría } & \multicolumn{2}{|c|}{ Hembras $(n=266)$} & \multicolumn{2}{|c|}{ Machos $(n=34)$} & \multirow{2}{*}{ Sig. } \\
\hline & & FA & FR & FA & FR & \\
\hline \multirow[t]{4}{*}{ Forma de los cuernos } & Cortos & 70 & 26.32 & 14 & 41.18 & $*$ \\
\hline & Curvos & 168 & 63.16 & 9 & 26.47 & $*$ \\
\hline & Espiral & 14 & 5.26 & 11 & 32.35 & $*$ \\
\hline & Intermedios & 14 & 5.26 & 0 & 0 & ns \\
\hline \multirow[t]{2}{*}{ Mamellas o zarcillos } & Presencia & 39 & 14.66 & 5 & 14.71 & ns \\
\hline & Ausencia & 227 & 85.34 & 29 & 85.29 & ns \\
\hline \multirow[t]{3}{*}{ Línea dorso-lumbar } & Recta & 126 & 47.37 & 18 & 52.94 & ns \\
\hline & Cóncava & 6 & 2.26 & 2 & 5.88 & ns \\
\hline & Convexa & 134 & 50.38 & 14 & 41.18 & ns \\
\hline \multirow[t]{3}{*}{ Posición de las orejas } & Horizontal & 6 & 2.26 & 9 & 26.47 & $*$ \\
\hline & Vertical & 0 & 0 & 0 & 0 & ns \\
\hline & Colgando & 260 & 97.74 & 25 & 73.53 & $*$ \\
\hline \multirow[t]{3}{*}{ Inclinación de la grupa } & Recta & 177 & 66.54 & 2 & 5.89 & $*$ \\
\hline & Poco inclinada & 75 & 28.19 & 15 & 44.11 & $*$ \\
\hline & Muy inclinada & 14 & 5.26 & 17 & 50.00 & $*$ \\
\hline \multirow[t]{3}{*}{ Angulosidad corporal } & Delgado & 81 & 30.45 & 8 & 23.53 & ns \\
\hline & Intermedio & 115 & 43.23 & 12 & 35.29 & ns \\
\hline & Ancho & 70 & 26.32 & 14 & 41.18 & $*$ \\
\hline \multirow[t]{4}{*}{ Capas de color } & Una capa & 9 & 3.38 & 5 & 14.71 & $*$ \\
\hline & Dos capas & 90 & 33.83 & 14 & 41.18 & ns \\
\hline & Tres capas & 137 & 51.50 & 8 & 23.53 & $*$ \\
\hline & Cuatro capas & 30 & 11.28 & 7 & 20.59 & ns \\
\hline
\end{tabular}

FA: frecuencia absoluta, FR: frecuencia relativa. ${ }^{*} \mathrm{p}<0.05$, ns: no hay diferencias estadísticamente significativas.

Los resultados del análisis de correspondencia múltiple mostraron que las variables que se asocian en ambas dimensiones, en orden de importancia de acuerdo con su magnitud, son la inclinación de la grupa $(0.87$ - 0.70) y la angulosidad corporal $(0.90-0.76)$. Sin embargo, la forma de los cuernos fue una variable que también presentó una magnitud importante en ambas dimensiones, pero sus valores fueron inferiores comparado con las dos variables mencionadas inicialmente para este análisis (0.19-0.15). Con respecto a este último caso, la línea dorso-lumbar fue la única variable que presentó un valor cercano a esta, pero solo en la primera dimensión (0.15). La varianza total explicada por las dimensiones uno y dos fue del $30.9 \%$ y el $25.5 \%$, respectivamente (Tabla 5 ).

Tabla 5. Matriz de discriminación obtenida con el análisis de correspondencia múltiple para los caracteres cualitativos de los 300 individuos

\begin{tabular}{cccc}
\hline Variables & Dimensión 1 Dimensión 2 & Media \\
\hline Forma de los cuernos & 0.185 & 0.145 & 0.165 \\
Mamellas o zarcillos & 0.023 & 0.010 & 0.017 \\
Línea dorso-lumbar & 0.146 & 0.023 & 0.084 \\
Posición de las orejas & 0.013 & 0.009 & 0.011 \\
Inclinación de la & 0.871 & 0.704 & 0.788 \\
grupa & 0.902 & 0.764 & 0.833 \\
Angulosidad corporal & 0.020 & 0.062 & 0.041 \\
Capas de color & 30.855 & 25.540 & 27.697 \\
\% de varianza & & &
\end{tabular}

Alfa de Cronbach promedio $=0.565$.

\section{DISCUSIÓN}

En este estudio se presentan los primeros resultados de caracterización morfológica para la cabra Motilona de la región Norte de Santander, donde se pudo observar que, las variables cuantitativas y cualitativas medidas en estos 300 individuos presentaron un importante grado de homogeneidad entre individuos. De acuerdo con estos resultados, la cabra Motilona pudiera ser definida como una raza autóctona de la región de estudio porque el grado alto de homogeneidad es evidencia de una población aislada que se adaptó a un sistema de producción bajo condiciones ambientales de una región geográfica especifica (7). En relación con esto, el CV es un parámetro descriptivo de los datos que permite determinar la homogeneidad de una población, donde valores de CV inferiores a $50 \%$ indican presencia de homogeneidad, pero valores cercanos a $20 \%$ y $5 \%$ indican homogeneidad moderada y alta, respectivamente (16). Por esta razón, se puede afirmar que los rasgos cuantitativos medidos en la cabra Motilona presentan un grado de homogeneidad moderado a alto. Según SilvaJarquin et al (7), se puede afirmar que existe homogeneidad en las variables morfológicas de una población de cabras si el CV no excede el valor de 30, como ocurrió en el caso de las hembras 
de este estudio. Sin embargo, en otro estudio realizado con ovinos, se argumenta que variables morfológicas con valores de CV superiores a 24 indican ausencia de homogeneidad (17). En este sentido, para el caso de las variables medidas en los machos se observó que el CV de todos los rasgos se encuentran dentro de los límites de homogeneidad, excepto el índice ICOMP (28\%). El alto valor de CV para este índice pudo deberse a que el peso de los machos tuvo una alta variación y en consecuencia afectó al rasgo ALCR. En relación con esto, Castellaro et al (3) argumentan que es normal encontrar estas variaciones en el peso de los machos debido por un lado a factores ambientales como la alimentación o las condiciones climáticas en donde son criados los animales y, por otro lado, a los genes de los propios individuos.

Los índices corporales utilizados en este estudio fueron utilizados para explorar las características raciales y las características funcionales de la población bajo estudio. De acuerdo con lo descrito por Abarca-Vargas et al (18), los índices raciales ICO, ITO, ICE e IPE definen a la cabra Motilona como un animal brevilíneo, longilíneo, dolicocéfalo y concavilíneo. Con respecto a las características funcionales $(3,18)$, los índices corporales IPRO, IMETO, IMCOS e IPEL indican que la cabra Motilona es un animal que presenta características morfológicas para la producción de carne, mientras que los índices corporales IPRP, IPET e ICOMP, la relacionan con una estructura corporal apta para la producción de leche. Estos resultados se deben a que la cabra Motilona es utilizada para ambos propósitos y por esta razón estos índices corporales no la clasifican como un animal de un único propósito.

En cuanto a los rasgos cualitativos, se observó que en la población estudiada predominaron hembras con cuernos curvos, sin mamellas, con orejas largas que cuelgan, con grupa recta, de angulosidad corporal intermedia a delgada y con tres capas de color de pelo diferentes. En los machos predominaron los rasgos de cuernos cortos, sin mamellas, orejas similares a las hembras, grupa muy inclinada, angulosidad intermedia a gruesa y con dos capas de color diferente de pelo. Estos resultados confirman lo reportado por otros estudios $(3,7)$, donde se ha observado que los machos tienden a ser más grandes, robustos y pesados que las hembras. Por esta razón, las variables peso, DB y ALCR, el tipo de cuernos y la longitud de las orejas parecen ser claramente variables discriminantes en una población de caprinos como lo afirman Gutiérrez et al (19).

Con este estudio se concluye que la cabra Motilona puede ser definida como un animal de tipo eumetrico y brevilíneo empleado para la producción de carne y leche en la región de Norte de Santander. A pesar de evidenciar un importante grado de homogeneidad en los rasgos morfológicos estudiados, es importante conocer también la relación con las características productivas porque de ese modo la cabra Motilona pudiera ser utilizada en programas de mejoramiento animal. Por lo tanto, futuros estudios en este aspecto son necesarios para profundizar en el conocimiento de las características morfológicas y productivas de la cabra Motilona de Norte de Santander.

\section{Conflicto de intereses}

Los autores declaran que no existe conflicto de intereses.

\section{Agradecimientos}

Los autores agradecen a la asociación Capriovinor de Cúcuta en Norte de Santander por facilitar el acceso a las diferentes granjas de donde se recolectaron los datos para este estudio.

\section{REFERENCIAS}

1. ICA. Censo pecuario nacional. Instituto Colombiano Agropecuario: Colombia; 2020. https://www.ica.gov.co/areas/pecuaria/ servicios/epidemiologia-veterinaria/ censos-2016/censo-2018.aspx
2. Ospina O, Montoya A, Montoya I, Grajales H. Reflexiones sobre la gestión de conocimiento y los sistemas de producción ovina y caprina en Colombia. Veterinaria y Zootecnia. 2014; 8(2):1-14. https://doi.org/10.17151/ vetzo.2014.8.2.1 
3. Castellaro G, Orellana C, Escanilla JP, Ruz Y. Características morfo-estructurales de un rebaño caprino de la zona mediterránea central de Chile. Agro Sur. 2020; 47(2):1929. https://doi.org/10.4206/agrosur.2019. v47n2-03

4. Hoffmann I, Scherf B. Implementing the Global Plan of Action for Animal Genetic Resources. Anim Genet Res. 2010; 47:1-10. https://doi.org/10.1017/ $\underline{\text { S2078633610001050 }}$

5. Hassen $H$, Baum M, Rischkowsky $B$, Tibbo M. Phenotypic characterization of Ethiopian indigenous goat populations. Afr J Biotechnol. 2012; 11(73):13838-13846. https://doi.org/10.5897/AJB12.2092

6. Boujenane I, Derqaoui L, Nouamane G. Morphological differentiation between two Moroccan goat breeds. J Livest Sci Technol. 2016; 4(2):31-38. https://doi. org/10.22103/JLST.2016.1515

7. Silva-Jarquin JC, Román-Ponce SI, DuránAguilar M, Vera-Ávila HR, CambrónSandoval VH, Andrade-Montemayor HM. Morphostructural Characterization of the Black Creole Goat Raised in Central Mexico, a Currently Threatened Zoogenetic Resource. Animals. 2019; 9(7):459. https://doi. org/10.3390/ani9070459

8. Solís-Lucas LA, Lanari MR, Oyarzabal MI. Caracterización fenotípica de la población caprina de la provincia de Santa Elena, Ecuador. Arch Zootec. 2020; 69(265):22-29. https://doi.org/10.21071/az.v69i265.5035

9. Peña S, López GA, Abbiati NN, Género ER, Martínez RD. Caracterización de ovinos Criollos argentinos utilizando índices zoométricos. Arch Zootec. 2017; 66(254):263-270. https://doi.org/10.21071/az.v66i254.2331

10. Barragán RM, Vargas DA, Ruiz VA, Rivera JH. Caracterización de la cabra criolla en la sub-provincia Volcanes de Colima y su sistema de producción. Arch Zootec. 2019; 68(263):332-341. https://doi. org/10.21071/az.v68i263.4190

11. Oseni SO, Ajayi BA. Phenotypic characterization and strategies for genetic improvement of WAD goats under backyard systems. Open J Anim Sci. 2014;4(5):253262. https://www.scirp.org/journal/ paperinformation.aspx?paperid $=50125$
12. Rafter JA, Abell ML, Braselton JP. Multiple comparison methods for means. Siam Review. 2002; 44(2):259-278. https:// epubs.siam.org/doi/pdf/10.1137/ $\underline{\mathrm{S} 0036144501357233}$

13. Kaiser HF. The varimax criterion for analytic rotation in factor analysis. Psychometrika. 1958; 23(3):187-200. http://128.174.199.77/psychometrika highly cited articles/kaiser 1958.pdf

14. Morrison DG. On the interpretation of discriminant analysis. J Mark Res. 1969; 6(2):156-163. https://journals.sagepub.com/ doi/abs/10.1177/002224376900600203

15. Di Franco G. Multiple correspondence analysis: one only or several techniques?. Qual Quant. 2016; 50(3):12991315. https:// doi.org/10.1007/s11135-015-0206-0

16. Rustom JA. Unidad 1 Estadística Descriptiva. Editor: Calandra P. En: Estadística descriptiva, probabilidad e inferencia. Una visión conceptual y aplicada. Edición 1. Universidad de Chile, Departamento de Economía Agraria: Chile; 2012. http://repositorio. uchile.cl/bitstream/handle/2250/120284/ Rustom Antonio Estadistica descriptiva.pdf

17. Flórez JM, Hernández MDJ, Bustamante MDJ, Vergara OD. Caracterización morfoestructural de tres poblaciones de Ovino de Pelo Criollo Colombiano OPC. Arch Zootec. 2018; 67(259):340-348. https:// doi.org/10.21071/az.v67i259.3789

18. Abarca-Vargas $D$, Macedo-Barragán $R$, Arredondo-Ruiz V, Valencia-Posadas M, Ayala-Valdovinos MA, Hernández-Rivera JA. Análisis de la morfología de la cabra mestiza de la subprovincia fisiográfica Volcanes de Colima, México. Rev Inv Vet Perú. 2020; 31(3):13. http://dx.doi.org/10.15381/ rivep.v31i3.16935

19. Gutiérrez RS, Luna RG, Nájera MDJF. Caracterización morfológica de un rebaño de conservación de cabras criollas en Zacatecas, México. Arch Zootec. 2018; 67(257):73-79. https://doi.org/10.21071/ az.v67i257.3493 